\title{
Integrating Big Data with Medical Imaging
}

\author{
Rasdianah, Fabian N.J. Souisa, Iwan Kurniawan Subagja, Budi Tri Rahardjo, Wenny \\ Anggeresia Ginting, K. Shankar, Phong Thanh Nguyen
}

\begin{abstract}
: it can consider that big data is massive amount of information that work wonderfully. Due to great potential that it has from last two decades this topic is taking special interest. In any industry the way in which it leverage, manage and analyze can change. The most promising field in which big data use for making the change is healthcare industry. For health-care and biomedical informatics research it increasingly used the technologies of big data. For certain advanced data analytics methods, predictive analytics and user behavior analytics the term big data used. At an unprecedented scale and speed huge amount of clinical and biological data have been collected and generated. For big data in the industry of healthcare the several sources incorporate medical examinations results, hospital records, devices and patients medical records that are a piece of internet of things. In medical imaging it also finds its usages. In order to treat, diagnose or monitor medical conditions to view the human body the various technologies that are used referred as medical imaging.
\end{abstract}

Keywords: Big data, Medical imaging, healthcare, technologies.

\section{INTRODUCTION}

The concept of big data is an ecosystem and new paradigm in the domain of biomedical that change case based studies into large scale. The big data characteristics are defined by 3 main features like velocity, volume and variety. It is called as $3 \mathrm{Vs}$ [1].

In any industry the way in which it leverage, manage and analyze can change. The most promising field in which big data use for making the change is healthcare industry. In general to avoid preventable diseases, improve life quality, predict outbreaks of epidemics and to reduce costs of treatment the medical analytics have the potential [2].

In medicine and healthcare the big data is referred as complex and large data, with suing of previous hardware or software they are difficult to analyze [3] [4]. Analysis, validation, data quality control, modeling, interpretation and integration of heterogeneous data cover by big data analytics [5]. From the available large amount of data it provides comprehensive knowledge discovering by big data analytics applications.

Revised Manuscript Received on September 22, 2019

* Correspondence Author

Rasdianah, Universitas Megarezky, Indonesia.

Fabian N.J. Souisa, Politeknik Perikanan Negeri Tual, Indonesia

Iwan Kurniawan Subagja, Universitas Krisnadwipayana Jakarta, Indonesia.

Budi Tri Rahardjo, Universitas Krisnadwipayana Jakarta, Indonesia.

Wenny Anggeresia Ginting, Universitas Prima Indonesia, Medan, Indonesia.

K. Shankar, Department of Computer Applications, Alagappa University, Karaikudi, India.

Phong Thanh Nguyen*, Department of Project Management, Ho Chi Minh City Open University, Vietnam. E-mail: phong.nt@ou.edu.vn
In medical imaging big data is very useful. In medical imaging it also finds its usages. In order to treat, diagnose or monitor medical conditions to view the human body the various technologies that are used referred as medical imaging. To designate the techniques set that produce the image of internal part of body the medical imaging is often used. For clinical purposes like dysfunction, examine injury, seeking to reveal, pathology or diagnose to create the images processes and techniques are used [6].

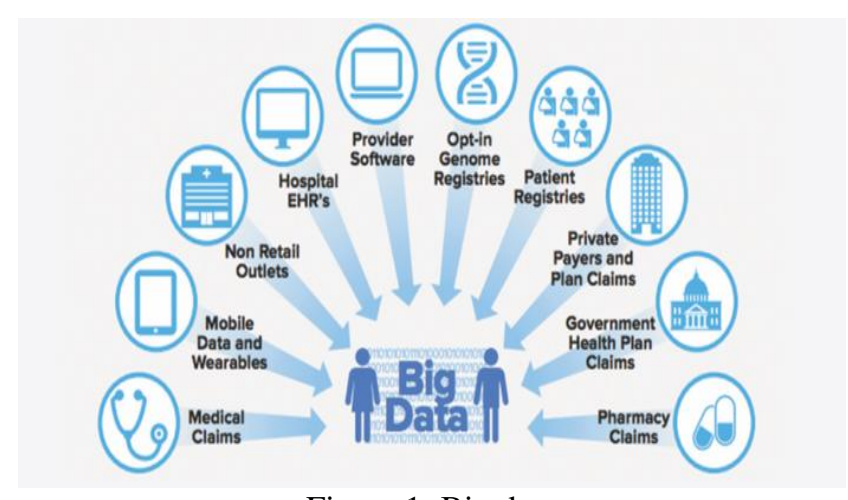

Figure 1: Big data

\section{II. MEDICAL IMAGING}

For the purpose of treatment and diagnostic to image the body of human several processes and modalities encompasses by medical imaging. In this way for all the people to improve the health of public it plays a significant role. In the follow up of a disease that is already diagnosed or treated the medical imaging is justified frequently [7].

Within digital health for treatment and diagnostic purpose the processes and techniques that used to create the images of several different human body parts referred as medical imaging. Several radiological imaging techniques include in medical imaging like:

1. Thermography

2. Elastography

3. Fluoroscopy

4. Tactile imaging

5. Endoscopy

6. X-ray radiography

7. MRI (Magnetic resonance imaging)

8. Technique of nuclear medicine functional imaging and medical photography

9. Ultrasound or medical ultrasonography [8] 


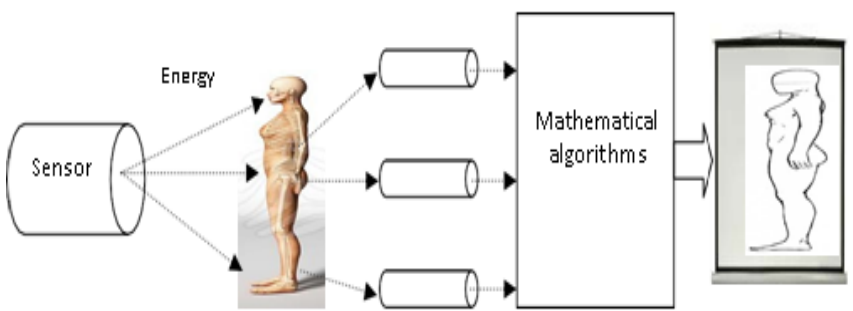

Figure 2: Concept of a medical imaging system

\section{IN DIAGNOSTIC MEDICA IMAGING BIG DATA CHARACTERISTICS}

In diagnostic imaging in the configuration of big data the five main characteristics that should be respected are as follows:

1. Value. To analysis of their coherent referred as value of big data. For clinicians and patients it should be valuable.

2. Variety. Heterogeneity and Complexity of several dataset that can be unstructured, structured and semi-structured known as variety.

3. Velocity. To the frequency and speed of data analysis, creation and processing and motion of data referred as velocity. Velocity refers to data in motion as well as and to the speed and frequency of data creation, processing and analysis. Consistently, a large number of patients experience analytic imaging techniques, which comprise of a reasonable work process in which data is obtained through an imaging gadget, put away in a PACS (standard picture archiving and communication) framework, and along these lines outwardly assessed on DICOM (digital imaging and communications in medicine) or PACS watcher by a specialist, who delivers an unstructured or structured report agent of the clinical result of the assessment [10].

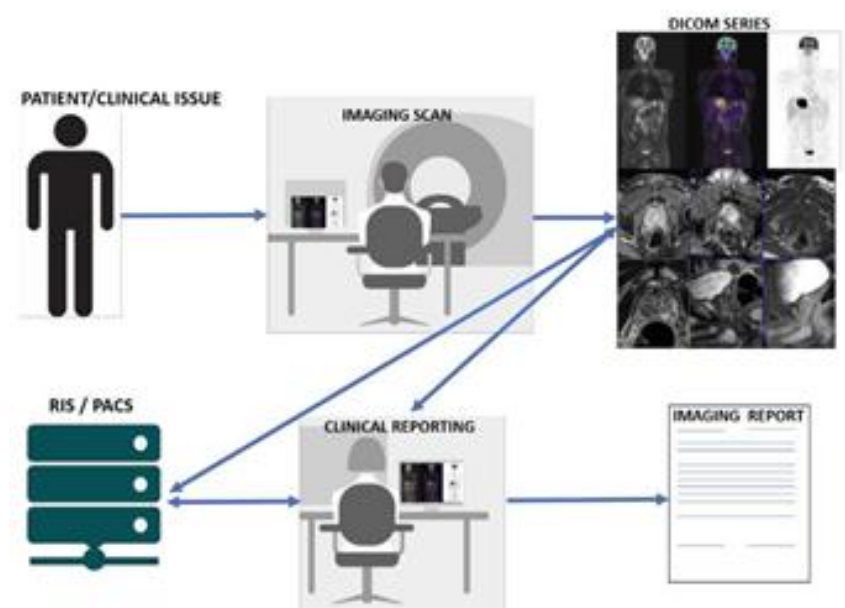

Figure 3: Workflow of Diagnostic Imaging

In a RIS (radiological information system) and PACS (standard picture archiving and communication) through an imaging device it can acquired the diagnostic images and therefore in it is inspected visually.

4. Veracity. Reliability, data quality, predictive value, uncertainty and relevance referred as veracity. Because decisions of death or life depend on the information reliability in healthcare data quality and Veracity are the fundamental issues.

5. Volume. In healthcare the quantity of big data referred as volume, which is assessed to drastically increment until the request for zettabytes (1021) by 2020 [9].

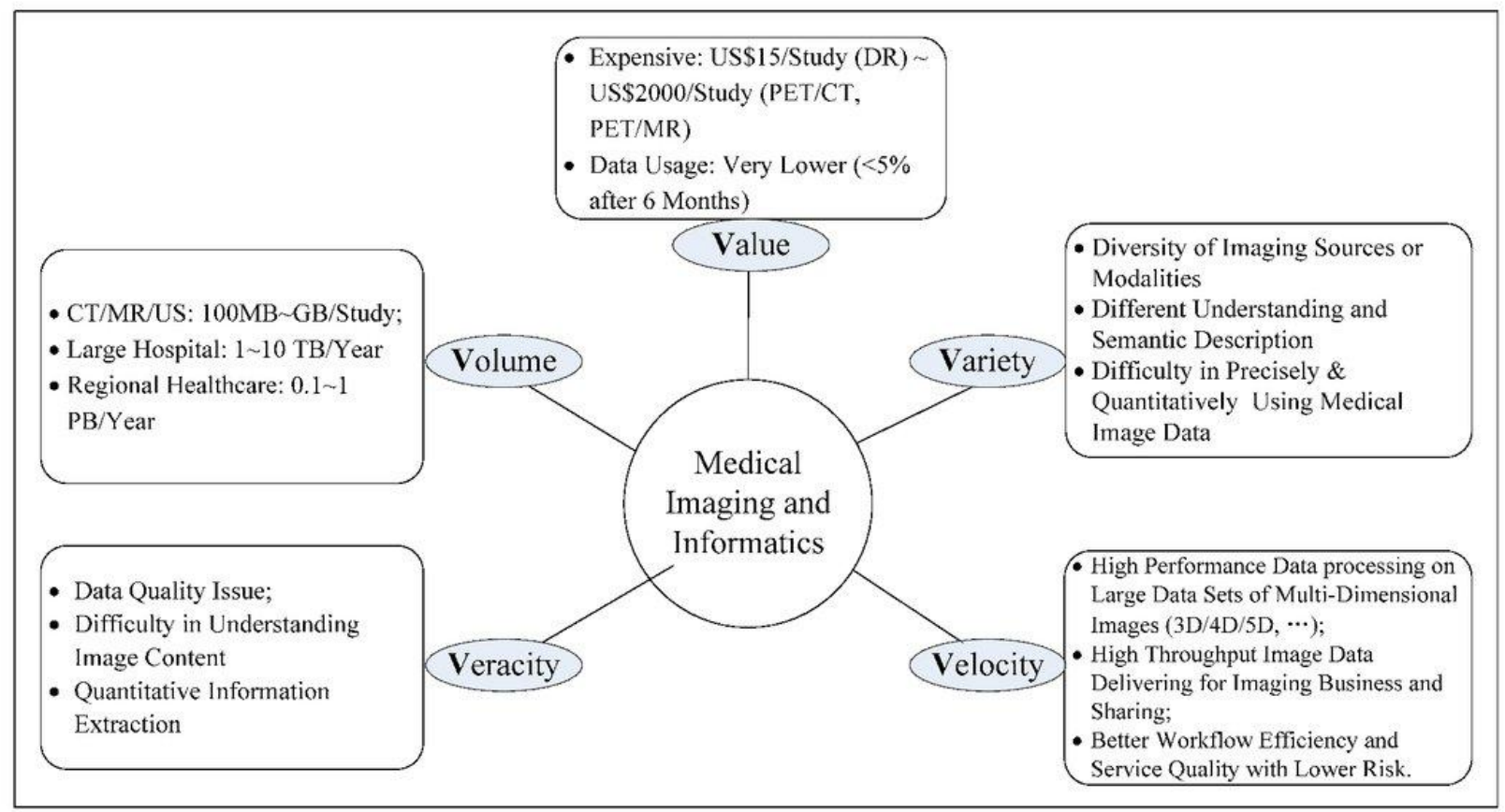

Figure 3: In medical imaging five v's of big data 


\section{MEDICAL IMAGING TECHNIQUES AND TYPES}

For imaging there exists several medical imaging types and as the technology being advance many more are being invented continuously. Some medical imaging techniques and types are described as follows:

1. Nuclear medicine: In radiology and nuclear medicine the radiation is used. Radiation is used in nuclear medicine and radiology. The radioactive materials are known as radiopharmaceuticals or radioisotopes in nuclear medicine; into the body they are introduced. From outside X-rays enter the body in radiology. It can consider that nuclear medicine is an imaging modality that includes inhalation, injection, or radioactive tracer's injection to several organs visualization. Through addition of a radioactive isotope to a pharmaceutical explicit to the body part being imaged the radiopharmaceutical or tracer id prodded. Gama radiation emits by radioactive tracer, by using gamma camera that can be imaged. [14].

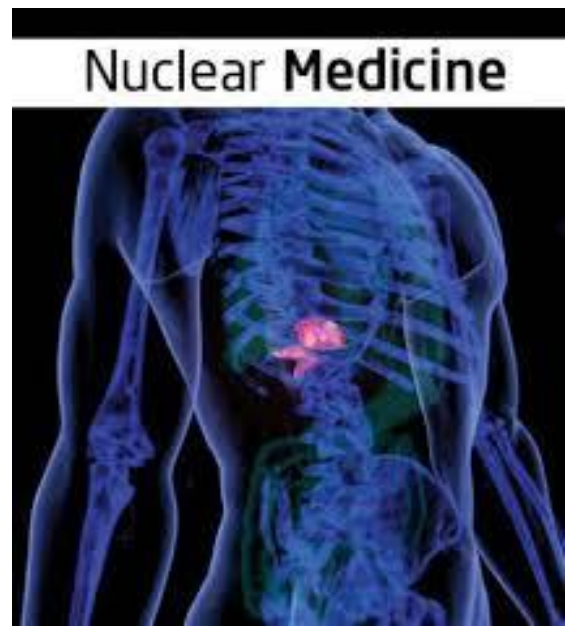

Figure 4: nuclear medicine

2. Ultrasound: From the inside of the body to capture live images high frequencies sound waves are using by a medical test that is known as ultrasound. Ultrasound is also called as sonography. The technology that is used in this system is same as the radar and sonar that are used to detect the ships and planes in military. To detect the problem with tissues, organs and vessels without need to create and incision the ultrasound helps the doctors. As compare with other methods of imaging it do not use radiation. In this way during pregnancy to watch the developing fetus this is a most preferred method [15].

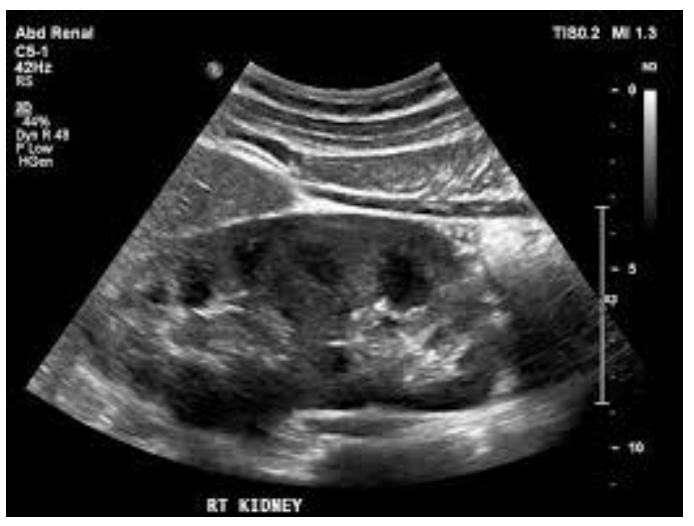

Figure 5: nornmal ultrasound image.

3. Magnetic resonance imaging (MRI): To form the physiological processes and pictures of the anatomy of the body the MRI consider as technique of medical imaging that used in radiology. In the body the scanners of MRI use magnetic field gradients, radio waves and strong magnetic fields to generate the images. The ionizing radiation or X-rays is not used in MRI, this is the reason it is different from PET, CAT or CT scans. It is consider as medical application of NMR (nuclear magnetic resonance) to magnetic resonance imaging. In other applications of NMR for imaging like NMR spectroscopy it can also used [13].

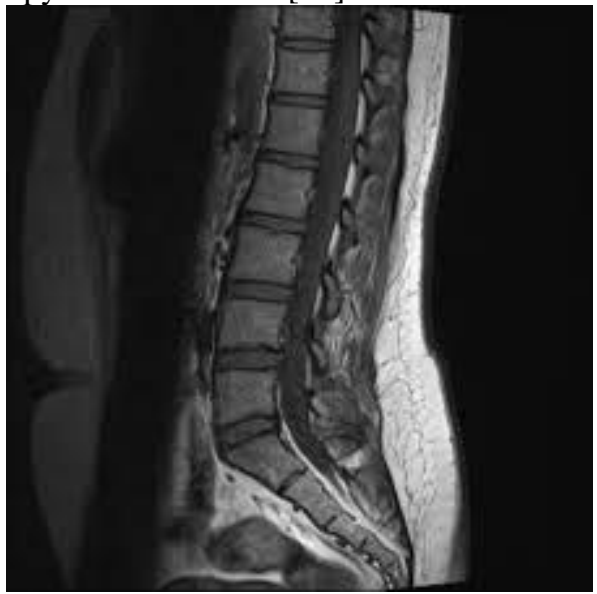

Figure 6: MRI of the spine

4. Radiography: To take the images of inside the body the electromagnetic radiation are used in Radiography. X- ray is the most common and well known type of radiography. On the body high energy waves beams by an x-ray machine in the procedure. Hard tissues like bones can absorb the wave but soft tissues like organs and skin cannot absorb these waves. The part of the body that absorbs the wave showing as a white on a film that transfers as a result of machine. The unabsorbed materials show as the black part[12].

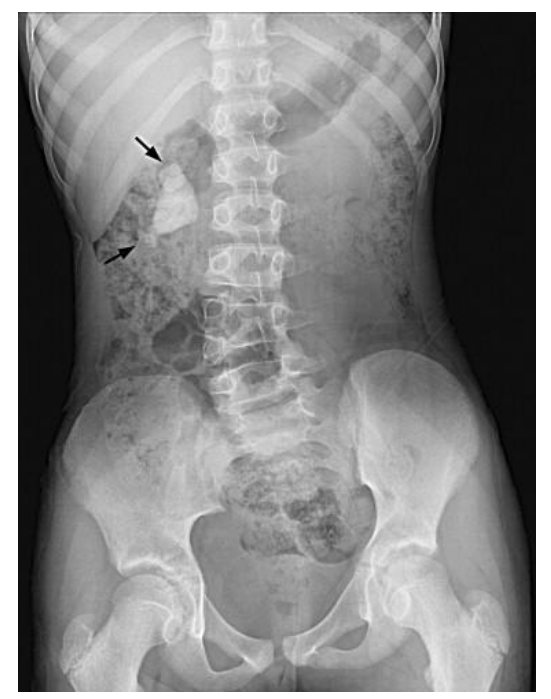




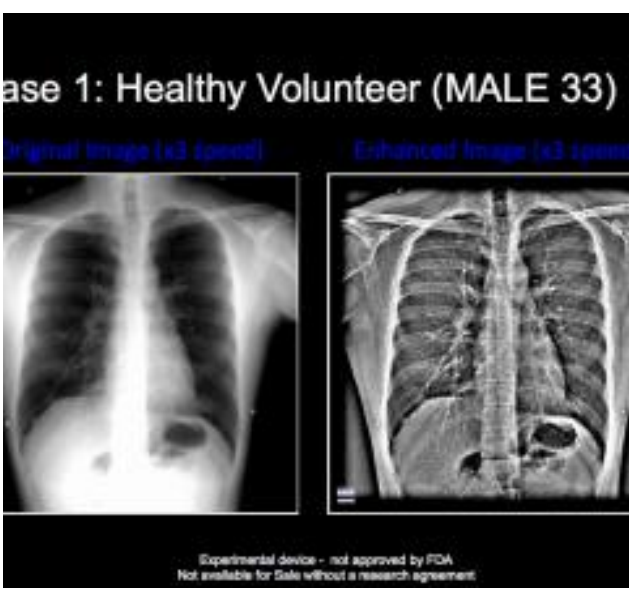

Figure 7: (1) overview of Radiography (b) digital Radiography

\section{FROM BIG DATA POINT OF VIEW USE OF MEDICAL IMAGE PROCESSING}

In addition to detecting diseases states on organ function and anatomy the medical imaging provides important information. Additionally, it is used for artery stenosis detection, identifying tumors in lungs, organ delineation, aneurysm detection, spinal deformity diagnosis etc. In the greater use of decision support systems in clinical settings and computer aided medical diagnostics he fast development in the quantity of health care organizations just as the quantity of patients has brought. By using computational intelligence in health care many areas can be improved like screening, diagnosis and prognosis [16]. To improve the diagnostic accuracy with appropriate care the integration of medical images helps [17]. With genomic data and other types of EHR (electronic health record) data the integration of medical images reduce the diagnosis time and improve the accuracy [18].

\section{CONCLUSION}

For big data in the industry of healthcare the several sources incorporate medical examinations results, hospital records, devices and patients medical records that are a piece of internet of things. In medical imaging it also finds its usages. In order to treat, diagnose or monitor medical conditions to view the human body the various technologies that are used referred as medical imaging. In any industry the way in which it leverage, manage and analyze can change. The most promising field in which big data use for making the change is healthcare industry. In medicine and healthcare the big data is referred as complex and large data, with suing of previous hardware or software they are difficult to analyze. For the purpose of treatment and diagnostic to image the body of human several processes and modalities encompasses by medical imaging.

\section{REFERENCES}

1. Jake Luo, Min Wu, Deepika Gopukumar \& Yiqing Zhao(2016), "Big Data Application in Biomedical Research and Health Care: A Literature Review".

2. Kankanhalli A, Hahn J, Tan S, Gao G. Big data and analytics in healthcare: introduction to the special section. Inform Syst Front. 2016;18:233-5
3. Kankanhalli A, Hahn J, Tan S \& Gao G. (2016)," Big data and analytics in healthcare: introduction to the special section". Inform Syst Front 2016, vol.18, pp.233-235.

4. Raghupathi W \& Raghupathi V(2014), "Big data analytics in healthcare: promise and potential". Health Inform Sci Syst, vol.2, issue.3.

5. Wu PY, Cheng CW, Kaddi CD, Venugopalan J, Hoffman R \& Wang MD(2017), " Omic and Electronic Health Record Big Data Analytics for Precision Medicine”. IEEE Trans Biomed Eng 2017, vol.64, pp. 263-273.

6. Wang Y, Kung LA, Wang WY, Cegielski CG. An integrated big data analytics-enabled transformation model: application to health care. Inf Manag. 2017;55:64-79.

7. Gligorijević V, Malod-Dognin N, Pržulj N. Integrative methods for analyzing big data in precision medicine. Proteomics. 2016;16:741-58.

8. Gaitanou P, Garoufallou E, Balatsoukas P. The effectiveness of big data in health care: a systematic review. In: Metadata and semantics research. 2014:141-53.

9. Dinov, I.D (2016), "Volume and Value of Big Healthcare Dat". J. Med. Stat. Inf. 2016, 4. pii: 3.

10. cMarco Aiello, Carlo Cavaliere, Antonio D'Albore \& Marco Salvatore(2019), "The Challenges of Diagnostic Imaging in the Era of Big Data". journal of clinical medicin, vol.8, issue. 316.

11. Archenaa J, Anita EM (2015), “ A survey of big data analytics in healthcare and government”. Procedia Comput Sci 2015, vol.50, pp.408-413.

12. Hermon R, Williams PA. Big data in healthcare: what is it used for?; Australian Ehealth Informatics and Security Conference; 2014. pp. 40-9.

13. Agarwal M, Adhil M, Talukder AK. International Conference on Big Data Analytics. Cham, Switzerland: Springer International Publishing; 2015. Multi-omics multi-scale big data analytics for cancer genomics; pp. 228-43.

14. Yao Q, Tian Y, Li PF, Tian LL, Qian YM, Li JS. Design and development of a medical big data processing system based on Hadoop. J Med Syst. 2015;39:23.

15. Ultrasound. https://www.healthline.com/health/ultrasound

16. H. Elshazly, A.T. Azar, A. El-korany \& A.E. Hassanien(2013), "Hybrid system for lymphatic diseases diagnosis". In International Conference on Advances in Computing, Communications and Informatics (ICACCI), pp.343-347, Aug 2013.

17. Geo Dougherty(2009), “ Digital image processing for medical applications". Cambridge University Press, 2009.

18. Ashwin Belle,S.M.Reza Soroushmehr, Fatemeh Navidi \& Raghuram Thiagarajan(2015),Big Data Analytics in Healthcare. Journal of Biomedicine and Biotechnology. 